\title{
Le Sonderforschungsbereich 980 : «Le savoir en mouvement. Transferts de savoir des mondes anciens jusqu'aux débuts de l'époque moderne »
}

Université Libre de Berlin

\section{Pierre Monnet}

\section{QpenEdition}

Édition électronique

URL : http://journals.openedition.org/ifha/8029

DOI : 10.4000/ifha.8029

ISSN : 2198-8943

Éditeur

IFRA - Institut franco-allemand (sciences historiques et sociales)

Édition imprimée

Date de publication : 31 décembre 2014

ISSN : 2190-0078

\section{Référence électronique}

Pierre Monnet, « Le Sonderforschungsbereich 980 : « Le savoir en mouvement. Transferts de savoir des mondes anciens jusqu'aux débuts de l'époque moderne » », Revue de l'IFHA [En ligne], 6 | 2014, mis en ligne le 31 décembre 2014, consulté le 20 avril 2019. URL : http://journals.openedition.org/ifha/8029 ; DOI : 10.4000/ifha.8029

Ce document a été généré automatiquement le 20 avril 2019

(CIFHA 


\section{Le Sonderforschungsbereich 980 : «Le savoir en mouvement. Transferts de savoir des mondes anciens jusqu'aux débuts de l'époque moderne »}

Université Libre de Berlin

Pierre Monnet

http://www.sfb-episteme.de/

Responsable : Gyburg Uhlmann

Ce programme de recherche porté depuis 2012 par l'Université libre de Berlin et associant l'Institut-Max-Planck d'histoire des sciences (Max-Planck Institut für Wissenschaftsgeschichte )$^{1}$ et le Musée des arts islamiques de la Fondation des Musées d'État de Berlin (Stiftung Preussischer Kulturbesitz, Museum für islamische Kunst) ${ }^{2}$, en coopération avec l'université Humboldt, s'intéresse aux mécanismes et processus de circulation et de mutation des savoirs dans les sociétés et les cultures européennes et non européennes jusqu'à la première modernité. La thématique entend ainsi souligner le caractère mobile et changeant des paramètres culturels et scientifiques dans des civilisations (Égypte ancienne, Islam classique, Moyen Âge...) réputées stables du point de vue des grands schèmes culturels. 20 sous-projets forment l'armature du programme chargé de débusquer, au-delà des instruments traditionnels de saisie du changement que sont les " césures ", les « révolutions ", les " progrès ", des évolutions souvent souterraines, faites de remploi, d'intégration et qui, ce faisant, échappent parfois au regard. Pour cela, le SFB choisit de privilégier un concept du savoir qui ne soit pas seulement la connaissance prise au sens large, mais définie comme une compétence scientifique qui s'érige comme telle et se révèle fondatrice de prescription, de légitimation et de valeur (ce qui inclut donc la prise en compte des institutions et des instances qui produisent et institutionnalisent tout ensemble le savoir). L'enquête privilégie ainsi dans tout acte de fixation, diffusion, 
autorisation, codification d'un processus de savoir non pas ce qui stabilise, mais au contraire fluidifie, met en mouvement, crée passage et lien. Trois lignes conductrices organisent la réflexion autour de la stratégie, de la tradition et de l'historicité du savoir en mouvement. Quatre groupes de recherche se sont constitués pour en partager l'analyse: celui sur "Transferts et les transculturalités", celui sur "Temps et historicité », celui sur « Représentation et médialité », celui sur "Structures et mutation du savoir ». Trois manières de classer le savoir et en même temps de l'étudier aujourd'hui dans ses formes historiques ont été détachées : «Dire », "Montrer », " Négocier ». Ces trois modalités permettent ainsi aux historiens de l'art, aux philologues, aux arabisants, aux spécialistes de la rhétorique, aux philosophes, aux théologiens ou aux historiens de la mondialisation de travailler ensemble.

2 Du terrain d'analyse ne sont pas exclues les modalités de diffusion et de transformation du savoir assurées aujourd'hui encore par les musées, dont les artefacts continuent de produire des effets d'interprétation, de fixation des connaissances qui participent de la notion de «savoir en mouvement ». C'est ainsi que le Musée des arts islamiques de Berlin conduit l'enquête sous cet angle sur ses propres objets dans la perspective d'un transfert de savoirs entre Moyen-Orient et Europe. Le SFB conçoit ou accompagne également des expositions dont le sujet entre directement dans le périmètre de son étude, telle celle sur les relations entre Inde, Perse et Europe à travers la figure de Joseph sous le titre « Joseph und Zulaikha. Beziehungsgeschichten zwischen Indien, Persien und Europa » du 11 juin au 7 septembre 2014.

3 Sur le plan plus académique, des projets spécifiques sont placés sous le toit de ce SFB, dont le libellé éclaire mieux encore les attendus de l'enquête, tels « La confection des listes dans l'Orient ancien" (multiscripturalité et multilinguisme, grammatologie et culture graphique) ou bien «Les transferts du savoir médical dans les œuvres encyclopédiques de l'antiquité tardive" (conservation, indexation, dénomination du savoir, en particulier dans la rencontre entre médecine talmudique et grecque), ou encore «La construction de la tradition à travers les commentaires du De interpretatione jusqu'au Moyen Âge ». Un ensemble de recherche en cours se déploie sur les interprétations et les réinterprétations croisées entre les traductions de la Bible et du Coran, y compris par le biais des translations grecques à Byzance, mais aussi sur le savoir des écrits apocryphes, sur les mutations de la mnémotechnique selon les lieux et les temps. Une grande attention est accordée à l'archéologie, non pas seulement comme science ou comme pratique, mais aussi comme un chaînon de la production et de la transformation du savoir par l'exhumation et la création de l'objet, lequel va devenir lui-même discours et support de parcours et de traces formant les linéaments d'un savoir en mouvement, jusqu'à son exposition. Les configurations du savoir sur le temps long sont également observées, de l'antiquité jusqu'aux XVIe et XVIIe siècles, ainsi des modes d'interrogation (poser et figurer, y compris graphiquement, une question), du merveilleux, du non-savoir, de la petitesse fausse, feinte, revendiquée, anoblie du presque-savoir (le « je ne sais quoi »).

4 Une série de conférences (Ringvorlesung) se met parallèlement en place, consacrée aux modes d'acquisition et d'expérimentation de nouveaux savoirs dans un contexte donné, tandis qu'un colloque annuel a réuni en juillet 2014 l'ensemble des chercheurs du SFB sous l'intitulé de l'«Itération comme transformation de la connaissance» (lecture, écriture, rituel, image, langage comme supports et facteurs de la répétition et de la transformation du savoir réitéré). En 2014, une première publication a ouvert la série des 
éditions issues de ce programme, celle de Pietro Daniel Omodeo, sous le titre Copernicus in the Cultural Debates of the Renaissance (Berlin : Brill, 2014).

\section{NOTES}

1. http://www.mpiwg-berlin.mpg.de/de/index.html. La Stiftung Preußischer Kulturbesitz est une fondation de droit public qui réunit et gère 19 musées, 15 collections, 5 instituts, ainsi que les archives et la Bibliothèque d'État de Berlin.

2. http://www.smb.museum/museen-und-einrichtungen/museum-fuer-islamische-kunst/ home.html\#.

\section{AUTEUR}

PIERRE MONNET

(EHESS/IFHA) 